\title{
1 Testing the adaptive walk model of gene evolution
}

2 Ana Filipa Moutinho ${ }^{*}, 1,2$, Adam Eyre-Walker ${ }^{2}$, Julien Y. Dutheil ${ }^{1,3}$

3

$4 \quad{ }^{1}$ Department of Evolutionary Genetics, Max Planck Institute for Evolutionary Biology, Plön, Germany

$5 \quad{ }^{2}$ School of Life Sciences, University of Sussex, Brighton, United Kingdom

$6 \quad{ }^{3}$ Unité Mixte de Recherche 5554 Institut des Sciences de l'Evolution, CNRS, IRD, EPHE, Université de Montpellier, Montpellier, France

9 *Corresponding author:

10 Ana Filipa Moutinho

11 Current address: School of Life Sciences, University of Sussex, Brighton, United Kingdom

12 E-Mail: a.f.moutinho@sussex.ac.uk

\section{Author Contributions:}

15 Conceptualization: AFM (equal), AEW (equal), JYD (equal)

16 Data curation: AFM (lead)

17 Formal analysis: AFM (lead)

18 Investigation: AFM (lead), AEW (contributing), JYD (contributing)

19 Methodology: AFM (equal), JYD (equal), AEW (contributing)

20 Project administration: JYD (lead)

21 Software: AFM (lead)

22 Supervision: JYD (lead)

23 Visualization: AFM (lead)

24 Writing - original draft: AFM (lead), JYD (contributing)

25 Writing - review \& editing: AFM (equal), AEW (equal), JYD (equal)

Preprint servers: bioRxiv (doi: https://doi.org/10.1101/2021.04.28.441765)

Classification: Major - Biological Sciences; Minor - Evolution, Genetics

Keywords: adaptive walk, molecular evolution, adaptive evolution, gene age, distribution of fitness

31 effects

This PDF file includes:

- Main text 
Understanding the dynamics of species adaptation to their environments has long been a central focus of the study of evolution. Theories of adaptation propose that populations evolve by "walking" in a fitness landscape. This "adaptive walk" is characterised by a pattern of diminishing returns, where populations further away from their fitness optimum take larger steps than those closer to their optimal conditions. Hence, we expect young genes to evolve faster and experience mutations with stronger fitness effects than older genes because they are further away from their fitness optimum. Testing this hypothesis, however, constitutes an arduous task. Young genes are small, encode proteins with a higher degree of intrinsic disorder, are expressed at lower levels, and are involved in species-specific adaptations. Since all these factors lead to increased protein evolutionary rates, they could be masking the effect of gene age. While controlling for these factors, we fitted models of the distribution of fitness effects to population genomic datasets of Arabidopsis and Drosophila. We found that a gene's evolutionary age significantly impacts the molecular rate of adaptation. Moreover, we observed that substitutions in young genes tend to have larger fitness effects. Our study, therefore, provides strong evidence that molecular evolution follows an adaptive walk model across large evolutionary timescale.

\section{Introduction}

How does adaptive evolution proceed in space and in time? This question has long intrigued evolutionary biologists. Fisher [1] proposed that adaptation relies on mutations with small effect sizes at the phenotypic level. He presented the geometric model of adaptation where phenotypic evolution occurs continuously and gradually towards an optimum fitness [1]. At the molecular level, Wright $[2,3]$ first introduced the idea that populations evolve in the space of all possible gene combinations to acquire higher fitness. He characterised this model of evolution as a "walk" in an adaptive landscape. Wright consequently proposed the shifting balance theory of adaptation, which combines the effects of drift and selection. Drift acts by moving the population away from its local peak, while natural selection directs the population to higher fitness, the so-called "global optimum" in the fitness landscape. With the rise of molecular genetics, Maynard Smith [4] extended this idea to a sequencebased model of adaptation. He introduced the idea of an "adaptive walk", where a protein "walks" in the space of all possible amino-acid sequences towards the ones with increasingly higher fitness values. Wright's and Maynard Smith's adaptation model was further extended by Gillespie [5-7], who presented the "move rule" in an adaptive landscape. Following Kimura's work on the effect sizes of mutations [8], Gillespie suggested that adaptation proceeds in large steps, where mutations with higher fitness effects are more likely to reach fixation. The adaptive walk model was later fully developed by Allen Orr [9,10], who extended Fisher's geometric model of adaptation and showed that adaptive walks lead to a pattern of diminishing returns. A sequence further away from its local optimum tends to accumulate large-effect mutations at the beginning of the walk, small-effect mutations being then only fixed when the sequence is approaching its optimum fitness. Therefore, under this model, 
adaptation relies both on mutations of large and small fitness effects, in a time-dependent manner. Experimental studies tracing the evolution of bacteria [11-14] and fungi [15] have supported this model. Experimental studies, however, can only assess patterns of adaptation at relatively short time scales in artificial environments. The challenge lies in studying adaptation across long evolutionary time scales: how does the distribution of beneficial mutations vary in time?

While long-term evolutionary processes are not directly observable, they leave a signature in genome sequences. Species share genes to variable extents, according to their degree of divergence. Some ancient genes, which evolved early, are shared by many species. Others, which evolved more recently, are only shared by a few related species $[16,17]$. Consequently, the age of a gene can be inferred from its phyletic pattern, that is, its presence or absence across the phylogeny [18]. These phyletic patterns are reconstructed using sequence similarity searches performed by tools like BLAST [19]. A gene is considered "old" if a homolog is identified in several taxa over a deep evolutionary scale, or "young" or lineage-specific if the recognised homologs are only present in closely related species. This approach is known as phylostratigraphy [20].

Previous studies suggested that young or lineage-specific protein-coding genes evolve faster than older ones [17,21-27]. Albà and Castresana [27] showed a negative correlation between the ratio of the non-synonymous $\left(d_{N}\right)$ to synonymous $\left(d_{S}\right)$ substitutions rates, $\omega$, and gene age in the divergence between humans and mouse, with young genes presenting a higher $\omega$. Cai and Petrov [22] reported similar findings using human-chimpanzee divergence data. By looking at polymorphism data, they further suggested that the faster evolution in young primate genes may be due to the lack of selective constraint posed by purifying selection and showed that these genes are more often positively selected. Similar correlations between $\omega$ and gene age have been observed in fungi [25], Drosophila [23,28,29], bacteria [30], viruses [31], plants [32,33], and protozoan parasites [34].

Despite the observed consistency across taxa, the underlying causal mechanisms remain debated [21]. As the average $d_{N} / d_{S}$ ratio integrates both positive and negative selection, a comparatively higher ratio can result from a less stringent purifying selection, the occurrence of positive selection, or both. Additionally, young and old genes differ in their structural properties, expression level, and protein function. Young genes tend to be smaller [22,24,35], have a higher degree of intrinsic disorder [36], and are expressed at lower levels [17,22,24,26]. Moreover, young genes tend to encode proteins involved in developing species-specific characteristics and immune and stress responses [16,37,38]. As the macromolecular structure [39,40], gene expression levels $[39,41]$ and protein function $[39,42,43]$ are known determinants of the rate of protein adaptation, they could be confounding the effect of gene age. Several studies reported the substantial impact of gene expression on the adaptive rate of proteins, where highly expressed proteins are significantly more constrained and have lower adaptation rates $[39,41,44,45]$. At the macromolecular level, some studies showed that highly disordered [39,40] and exposed residues [39] present higher rates of adaptive evolution. Finally, there is evidence that proteins involved in the immune and stress response have higher 
111 molecular adaptive rates $[39,43,46,47]$. Thus, controlling for these confounding factors when

112 assessing the impact of gene age on the rate of molecular adaptation is crucial.

113 Here, we used a population genomic approach to test the adaptive walk model. We make two

114 predictions: first, that younger genes are undergoing faster rates of adaptive evolution, and second, the

115 evolutionary steps they make are larger. We tested the first prediction by estimating rates of adaptive

116 and non-adaptive protein evolution using an extension of the MacDonald-Kreitman (MK) test [48],

117 which uses counts of polymorphism and substitution at selected and neutral sites. We quantified the

118 rates of adaptive and non-adaptive evolution using the statistics $\omega_{a}$ and $\omega_{n a}$, which denote the rates of

119 adaptive and non-adaptive non-synonymous substitution relative to the mutation rate. We investigated

120 whether protein length, gene expression, relative solvent accessibility (RSA), intrinsic protein

121 disorder, BLAST's false-negative rate, and protein function act as confounding factors of the effect of

122 gene age. To test the second prediction, we considered the rates of substitution between amino acids

123 separated by different physicochemical distances as a function of gene age. We tested our hypotheses

124 in two pairs of species with different life-history traits: the diptera Drosophila melanogaster and D.

125 simulans and the Brassicas Arabidopsis thaliana and A. lyrata. In each species pair, we compared their 126 most recent genes with those dating back to the origin of cellular organisms.

\section{Results}

129 We tested the adaptive walk model of sequence evolution by assessing the impact of gene age on the

130 rate of adaptive $\left(\omega_{a}\right)$ and non-adaptive $\left(\omega_{n a}\right)$ non-synonymous substitutions. To assess whether the

131 effect of gene age persisted when controlling for multiple confounding factors, we applied a non-

132 parametric measure of correlation between gene age and $\omega_{a}$ and $\omega_{n a}$ for each category of the co-

133 factors analysed. The overall effect of gene age on $\omega_{a}$ and $\omega_{n a}$ in each co-factor was assessed by

134 combining significance values across tests in both species using the weighted Z-method [49].

Young genes have a higher rate of adaptive substitutions

137 We tested the adaptive walk model of sequence evolution by assessing the impact of gene age on the 138 rate of adaptive $\left(\omega_{a}\right)$ and non-adaptive $\left(\omega_{n a}\right)$ non-synonymous substitutions. We found that gene age 139 significantly impacts estimates of $\omega, \omega_{a}$ and $\omega_{n a}$ in both species' pairs (Table 1 and Figure 1b). This 140 result suggests that the higher $\omega$ ratio of more recently evolved genes is due to a higher rate of 141 adaptive and non-adaptive non-synonymous substitutions. As X-linked genes are known to evolve 142 faster due to the male hemizygosity [50-52], we assessed whether the relationship between 143 evolutionary rates and gene age differed between chromosomes in Drosophila (Figure 1b). We

144 compared models with and without the chromosome's effect (see Material and Methods and 145 supplementary file S1) and found low support for a chromosomal effect ( $\mathrm{p}=0.041$ for $\omega_{n a}$ and $\mathrm{p}=$ 1460.094 for $\omega_{a}$ ). We, therefore, combined data from all chromosomes for subsequent analyses. 
The effect of gene age on the rate of molecular adaptation is robust to multiple confounding factors

Genes of different ages intrinsically differ in their features $[16,22,36]$. As such traits significantly impact the rate of molecular evolution [39], they may be confounding the faster adaptive rates observed in young genes. Here, we assessed whether the effect of gene age on the rate of molecular adaptation persisted after controlling for multiple confounding factors. To do so, we first assessed the correlation of gene age with the rates of molecular evolution in distinct categories of genes, according to a putative confounding factor. As estimates of the rate of adaptive substitutions for a small number of genes exhibit large sampling variances [53,54], we could only assess each confounding factor individually with this apporach.

Previous studies reported that younger genes encode shorter proteins $[24,35,55]$ and are expressed at lower levels $[17,22,24,26]$, a pattern that we also observed in our data set (gene age vs. protein length: Kendall's $\tau=-0.485, \mathrm{p}=2.82 \mathrm{e}-02 ; \tau=-8.48, \mathrm{p}=1.06 \mathrm{e}-05$, Figure S1a; gene age vs. gene expression: $\tau=-0.595, \mathrm{p}=7.35 \mathrm{e}-03 ; \tau=-0.790, \mathrm{p}=4.00 \mathrm{e}-05$, Figure $\mathrm{S} 1 \mathrm{~b}$ in supplementary data; for D. melanogaster and A. thaliana, respectively). As younger proteins are shorter than older ones, they have a higher proportion of exposed residues [39]: gene age is significantly positively correlated with the average RSA per gene $(\tau=0.636, \mathrm{p}=3.98 \mathrm{e}-03 ; \tau=0.695, \mathrm{p}=3.03 \mathrm{e}-04$, for $D$. melanogaster and $A$. thaliana respectively; Figure S2a in supplementary data). Because exposed residues are more flexible [56], young genes tend to encode proteins with a higher degree of intrinsic disorder, a pattern previously reported in mice [36]. We confirmed this pattern in D. melanogaster $(\tau=0.606, \mathrm{p}=6.10 \mathrm{e}$ 03; Figure S2b in supplementary data) and A. thaliana $(\tau=0.467, \mathrm{p}=1.53 \mathrm{e}-02$; Figure S2b in supplementary data).

We split our data into two roughly equal sized groups according to protein length, expression level, average RSA, and average intrinsic disorder and reran the analysis within the "high" and "low" groups, combining probabilities from the two analyses using the weighted Z-method [49]. Some phylostrata were further combined when under-represented in some gene categories (see Material and Methods). We found that $\omega, \omega_{n a}$ and $\omega_{a}$ remain significantly correlated to gene age, except when controlling for protein length and gene expression for $\omega_{a}$ in Arabidopsis (Figure 2 and Table 1). This weaker effect may be a consequence of how the most recent clades were combined in these analyses, as there was little data available for those genes (see Material and Methods). Nonetheless, when combining probabilities across the two species, we observed a significant correlation between all measures of evolutionary rate and gene age controlling each of the co-factors (Table 1).

We further jointly estimated the combined effect of the potential confounding factors by applying a recently developed method that extends the MK test with a generalized linear model [57]. This approach allows us to disentangle between direct and indirect effects on the rate of adaptive substitutions by analysing multiple factors simultaneously. However, this method does not account for segregating slightly deleterious mutations, which can bias estimates of the rate of adaptive 
allele frequency was below $50 \%$ to minimize any potential bias. Despite the large reduction in the data set, this analysis revealed a strong effect of gene age (Table S1 in supplementary data). Our findings, therefore, suggest that the effect of gene age on rates of protein evolution is robust to the tested confounding factors and that a gene's age acts as a significant determinant of the rate of adaptive and non-adaptive evolution in both species.

The effect of gene age on the molecular rate of adaptation is robust to BLAST's false negative rates The physlostratigraphy approach has been previously used to date the emergence of new genes, and some studies have pointed out its potential limitations [28,59-62]. Because BLAST homology searches might fail to identify homologs in short or rapidly evolving genes, such genes could be mistakenly classified as young. To assess whether BLAST's false negative rate could explain the correlation between gene age and the rate of adaptive evolution, we analysed the gene age's effect by correcting the variation in E-value estimates from BLAST's searches between each gene and their respective outgroups. As expected, we observed that genes in younger phylostrata present higher Evalues in both species $(\tau=0.564, \mathrm{p}=0.025 ; \tau=0.951, \mathrm{p}=1.20 \mathrm{e}-06$, for $D$. melanogaster and $A$. thaliana respectively; Figure S3a in supplementary data). To control this effect, we reran our analyses with a subset of genes for which the correlation between the E-value and gene age was no longer significant (see Material and Methods and supplementary file S2) $(\tau=0.408, p=0.111 ; \tau=0.354, p=$ 0.141, for D. melanogaster and A. thaliana respectively; Figure S3b in supplementary data). We observed that the effect of gene age prevailed for all estimates in the two species $(\omega: \tau=0.929, p=$ $1.30 \mathrm{e}-03 ; \omega_{n a}: \tau=0.786, \mathrm{p}=6.49 \mathrm{e}-03 ; \omega_{a}: \tau=0.643, \mathrm{p}=2.59 \mathrm{e}-02$ in A. thaliana; and $\omega: \tau=0.697, \mathrm{p}$ $=1.61 \mathrm{e}-03 ; \omega_{n a}: \tau=0.636, \mathrm{p}=3.98 \mathrm{e}-03 ; \omega_{a}: \tau=0.636, \mathrm{p}=3.98 \mathrm{e}-03$ in D. melanogaster, Figure S4 in supplementary data). These results suggest that the correlation of gene age with the rate of adaptive evolution cannot be attributed to errors in dating the emergence of a gene stemming from the failure of identifying homologs in older taxa.

\section{The effect of gene age on the rate of molecular adaptation does not depend on protein function}

Lineage-specific genes are known to be involved in species-specific adaptive processes, such as the evolution of morphological diversity [63] and immune and stress responses [17,34,63]. As proteins encoding such functions tend to have higher molecular rates of adaptation [39,42,43,46,47,64], we further assessed whether the observed effect of gene age could be due to younger genes being enriched in functions with higher evolutionary rates. We first examined which functions are encoded by young genes in A. thaliana and D. melanogaster. In A. thaliana, young genes (Clades 12 to 15 in Figure 1a) are mostly involved in a large variety of cellular processes, stress response and external stimulus, protein binding, and signal transduction (Figure S5a in supplementary data). In D. melanogaster, young genes (Clades 11 and 12 in Figure 1a) encode mostly functions involved in the cell's anatomic 
structure, stress response, nervous system processes, enzyme regulators, immune system mechanisms, and a wide range of metabolic processes (Figure S5b in supplementary data).

To further correct for the potential bias of protein function, we assessed the effect of gene age separately for several GO-annotated genes, when a sufficient number of annotated genes in each age class was available (see Material and Methods). In A. thaliana, we found that the impact of gene age on $\omega_{a}$ is stronger in proteins linked to stress response and cellular components, where younger genes present higher molecular adaptive rates (Figure S6a and supplementary file S3 in supplementary data). Although the GO term cellular component represents a comprehensive annotation, it denotes the cellular compartments where processes such as signal transduction and membrane trafficking occur, essential for maintaining the cell homeostasis $[65,66]$. In D. melanogaster, we observed a strong effect of gene age on $\omega_{a}$ for proteins encoding chromosomal organisation, protein complex, stress response, signal transduction, and involved in the cell cycle (Figure S6b and supplementary file S3 in

233 supplementary data in supplementary data). Even though these functions cover a wide range of 234 molecular processes, they are involved in DNA replication, genome stability, and immune and stress 235 responses, which are critical functions for the co-evolutionary arms race between hosts and parasites

236 [47]. When looking at $\omega_{n a}$, our analyses revealed a strong influence of gene age in most functions analysed in both species, where young genes present higher rates of non-adaptive substitutions (Figure

238 S6 and supplementary file S3 in supplementary data in supplementary data). These results suggest 239 that, when restricting the analysis to proteins involved in defence mechanisms, which are known to 240 adapt faster $[42,43,47,64]$, gene age still has an impact on the efficiency of selection acting upon a 241 protein.

\section{$\underline{\text { Substitutions in young genes have larger effect sizes }}$}

244 Our second prediction under the adaptive walk model is that substitutions in young genes should have 245 larger fitness effects than in older genes. To test this prediction, we used Grantham's physicochemical distances between amino-acids [67] as a proxy for the fitness effects of amino-acid substitutions. We

247 looked at the fixed differences separated by one mutational step between each pair of species and 248 reported the average Grantham's distances between residues within each age stratum. We observed 249 that substitutions in young genes tend to occur between less biochemically similar residues

250 (Arabidopsis: $\tau=1, \mathrm{p}=2.00 \mathrm{e}-07$; Drosophila: $\tau=0.788, \mathrm{p}=3.628 \mathrm{e}-04$; Figure 3 and supplementary 251 file S4), suggesting that substitutions in these genes have larger fitness effects than in old ones. To 252 further test whether these larger fitness effects had a positive or negative impact on younger genes, we 253 estimated the average Grantham's distance amongst adaptive $\left(\bar{G}_{a}\right)$ and non-adaptive $\left(\bar{G}_{n a}\right)$ non254 synonymous substitutions (see Material and Methods and supplementary file S4). We expect a 255 positive correlation between $\bar{G}_{a}$ and $\bar{G}_{n a}$ and gene age, i.e., the average Grantham's distance amongst 256 adaptive and non-adaptive substitutions should increase towards the present. This is indeed what we 257 find. We observed a positive correlation between $\bar{G}_{a}$ and gene age in both species, which is 
individually significant in Drosophila $(\mathrm{p}=0.006)$ and significant across species when we combine probabilities $(\mathrm{p}=0.009)$ (Figure $\mathrm{S} 7)$. We observed the same pattern in the analysis of $\bar{G}_{n a}$ with a significant correlation in Arabidopsis $(\mathrm{p}=0.004)$ and a significant correlation when combining the probabilities from both species $(\mathrm{p}=0.0007)$ (Figure $\mathrm{S} 7)$. These findings suggest that substitutions in young genes tend to be more adaptive and under less constraint, although the strength of selection differs between species.

\section{Discussion}

266 We used a population genomic approach to disentangle the effects of positive and negative selection on the rate of non-synonymous substitutions. Using complete genome data from two Arabidopsis and Drosophila species, we showed that the higher rate of non-synonymous substitutions in younger genes results both from relaxed purifying selection (higher $\omega_{n a}$ ) and a higher rate of adaptive substitutions (higher $\omega_{a}$ ) (Figure 1b). By looking at the magnitude effect of gene age, we observed that young genes present a 25-fold higher rate of adaptation than older genes in Drosophila species and around 30-fold higher in Arabidopsis. The magnitude of this effect is higher than that observed for recombination rate and solvent exposure in these species, two other factors strongly correlated to the rate of adaptive evolution $[39,68]$. We also observe that young genes undergo substitutions that are larger in terms of physicochemical properties than older genes. A question remains: what are the drivers of these effects?

The magnitude effect of gene age on adaptive evolution is species-specific

Although we observed a strong impact of gene age on the molecular adaptive rate in both species pairs, the shape of the relation between these two variables differs. While the relationship between gene age and $\omega_{a}$ is monotonously increasing in Arabidopsis, it has several peaks in Drosophila (Figure 1b). This pattern is particularly evident if we discard the two youngest clades. In Drosophila, the correlation becomes much weaker and non-significant for $\omega_{a}\left(\omega: \tau=0.600, \mathrm{p}=0.016 ; \omega_{n a}: \tau\right.$ $=0.556, \mathrm{p}=0.025 ; \omega_{a}: \tau=0.467, \mathrm{p}=0.060$ ), whereas, in Arabidopsis, the effect of gene age persists $\left(\omega: \tau=0.9487, \mathrm{p}=6.342 \mathrm{e}-06 ; \omega_{n a}: \tau=0.872, \mathrm{p}=3.345 \mathrm{e}-05 ; \omega_{a}: \tau=0.692, \mathrm{p}=9.86 \mathrm{e}-04\right)$. emergence of young genes in this species [17]. The peak in the adaptive substitution rate observed for clades 6 and 7 (Figure 1b) coincided with the animal phyla's major radiation at the time of extensive periods of glacial cycles [69]. When looking at the functions coded by these proteins, we found that they are linked to a wide range of vital cellular and biological processes, such as defence mechanisms and cell differentiation (Figure S8 in supplementary data). This pattern suggests that these genes might be experiencing higher molecular adaptive rates due to their role in such vital processes. However, for these genes to keep such high rates of adaptive substitutions until recent times (i.e., in the branch 
have proposed that functional epistasis is an important factor in the evolution of genes involved in defence mechanisms and adaptation to new environmental stresses [70-74]. We posit that such gene interactions keep these proteins further from their optimum throughout time due to the rugged shape of the fitness landscape, leading to the high molecular adaptive rates observed in the branch between $D$. melanogaster and D. simulans. To further test this hypothesis, we used the degree of protein-protein interactions (PPI) as a proxy for epistatic interactions and analysed its relationship with gene age. We observed that genes in clade 7 have a slightly higher degree of PPI than other strata (Figure S9 in supplementary data and supplementary file S5), suggesting that these genes might be experiencing relatively more epistatic interactions. These findings are consistent with epistasis influencing the evolution of these genes, potentially explaining their continued higher rates of molecular adaptation. In contrast, the burst of the emergence of new genes in Arabidopsis coincided with the plant-specific radiation right before the emergence of Brassicaceae $[17,75]$. This trend is consistent with our results from $A$. thaliana, where the bursts of $\omega_{a}$ occur in younger clades (after clades 11 and 12 in Figure $1 \mathrm{~b}$ ). These distinct patterns observed between species suggest that the role of a gene's age in molecular adaptation is complex, as also evidenced by the lack of a significant correlation with $\omega_{a}$ previously reported in humans [22]. The authors proposed that this result may be a consequence of the generally low molecular adaptive rates observed in primates $[22,48]$.

Despite these species-specific trends, our analyses revealed a strong correlation between $\omega_{a}$ and gene age extending through hundreds of millions of years (Figures 1 and 2). These findings suggest a consistent effect of a gene's age on the rate of molecular adaptation across taxa.

\section{An adaptive walk model of gene evolution}

317 Our study highlighted that, after their emergence, young genes evolve through relaxed selection, as

318 first proposed by Ohno [76], but also by acquiring beneficial mutations, as described in the "adaptiveconflict" model $[37,77]$. Ohno's idea of evolution was "non-Darwinian" in its nature, as he believed that "natural selection merely modified while redundancy created" [76]. He proposed that new genes evolve by accumulating "forbidden" mutations, where they are only preserved if the development of a formerly non-existent function occurs, a process known as neo-functionalisation. In this scenario, natural selection only acts at the stage of acquiring a new function. Further extensions of this theory suggested that the preservation of a new gene can also occur through sub-functionalisation, where the accumulation of deleterious mutations leads to a complementary loss of function in both copies of the gene [78,79].

In contrast, the "adaptive-conflict" model assumes that the ancestral gene could carry more than two pleiotropically constrained functions [37,77]. Once the duplication event occurs, each copy then becomes specialised in one of the ancestral functions. In this case, the ancestral gene's split proceeded through positive Darwinian selection [37,77]. These theories are based on the evolution of gene duplicates and agree with the idea of evolution as a "tinkerer" proposed by Jacob [80], where 
evolution adjusts the already existing elements. In de novo evolution, however, new genes emerge by acquiring new functions from the non-coding fragments of the genome $[17,81,82]$. This process is

334 thought to proceed through a stochastic phase followed by the successive accumulation of beneficial

335 mutations, ultimately leading to a new function with a species-specific selective advantage [83-86].

336 When looking at the fundamental ideas behind these theories, one can draw one prominent

337 feature that portrays the evolution of new genes: young genes are further away from their fitness

338 optimum. Hence, we posit that these genes follow an adaptive walk model of gene evolution to reach

339 their fitness peak $[3,87,88]$. As their full potential has yet to be met, more consecutive beneficial

340 mutations are theoretically needed to reach their fitness optimum, leading to the higher molecular

341 adaptive rates observed in these genes. In turn, older genes are closer to their optimal features and less

342 robust to large effects' mutations, thus only accumulating mutations with small fitness effects. Such

343 slightly advantageous mutations are more difficult to select for, leading to lower adaptive rates in

344 these proteins. We further tested this hypothesis using Grantham's physicochemical distances [67] as a

345 proxy for the fitness effect of substitutions. This analysis showed that substitutions in young genes

346 tend to occur between more dissimilar residues (Figure 3), suggesting that the evolution of young

347 genes proceeds in larger steps compared to old ones. Moreover, by looking at the proportion of

348 adaptive substitutions across Grantham's distances categories in each age class, we observed that

349 adaptive substitutions in young genes are under stronger positive selection (Figure S7 in

350 supplementary data). However, the strength of this signal differs between species. While we observed

351 a strong positive correlation between $\bar{G}_{a}$ and gene age in Drosophila, this relationship is only weak in

352 Arabidopsis. The opposite pattern is observed for the correlation between $\bar{G}_{n a}$ and gene age, where

353 this relationship is only significant in Arabidopsis (Figure S7 in supplementary data). These patterns

354 suggest that, in Arabidopsis, there is a stronger influence of relaxed purifying selection when

355 compared to positive selection. This weaker adaptive signal may be a consequence of their mating

356 system. The transition from out-crossing to self-fertilization in A. thaliana exposed these plants to less

357 efficient purifying selection due to their reduced effective population sizes [89,90], thus accumulating

358 more deleterious mutations. This higher load of segregating deleterious mutations can interfere with

359 nearby beneficial mutations, preventing their fixation and slowing down adaptation. Indeed, previous

360 studies have shown that segregating recessive deleterious mutations can substantially decrease the

361 fixation rate of advantageous mutations, leading to a weaker adaptive signal in some parts of the

362 genome [91,92]. In contrast, the large effective population sizes of Drosophila species make them

363 more efficient in removing deleterious mutations and prone to stronger positive selection [93-95].

364 These patterns could explain the observed differences in the strength of selection in young genes

365 between species. Overall, our findings support an adaptive walk model hypothesis of gene evolution

366 over time, where protein-coding genes adapt following a pattern of diminishing returns in Drosophila

367 and Arabidopsis. The increasing availability of population genomic datasets will allow to characterize

368 the specificities of the adaptive walk model of gene evolution in an increasingly diverse range of taxa. 


\section{Material and Methods}

We assessed the role of gene age on adaptive evolution using the divergence and polymorphism data published in Moutinho et al. [39]. For Drosophila melanogaster, the data included 10,318 proteincoding genes in 114 genomes for one chromosome arm of the two large autosomes $(2 \mathrm{~L}, 2 \mathrm{R}, 3 \mathrm{~L}$, and $3 R$ ) and one sex chromosome (X) pooled from an admixed sub-Saharan population from Phase 2 of the Drosophila Genomics Project [DPGP2] [96] and divergence estimates from D. simulans (Table S2 in supplementary data online). For Arabidopsis thaliana, we analysed 18,669 protein-coding genes in 110 genomes comprising polymorphism data from a Spanish population (1001 Genomes Project) [97] and divergence out to A. lyrata (Table S3 in supplementary data online). We used these data sets to infer the synonymous and non-synonymous unfolded site frequency spectrum (SFS), and synonymous and non-synonymous divergence from the rate of synonymous and nonsynonymous substitutions. Sites with a missing outgroup allele were considered as missing data. All estimates were obtained using the "bppPopStats" program from the Bio++ Program Suite [98]. Gene age data were obtained from published data sets, wherein 9,004 Drosophila [28] and 17,732 Arabidopsis [33] genes were used. Analyses were performed by dividing genes into 12 and 15 phylostrata for D. melanogaster and A. thaliana (Figure 1), respectively, numbered from the oldest (stratum 1) to the most recent (strata 12 and 15 in D. melanogaster and A. thaliana, respectively). The most recent clades include orthologous genes present in each species and their respective outgroups. The analyses on the X-linked and autosomal genes in D. melanogaster were performed with 1,478 and 7,526 genes, respectively. We fitted models of the distribution of fitness effects (DFE) across different age classes and gene categories to estimate the molecular rate of adaptation [48].

Estimation of the adaptive and non-adaptive rate of non-synonymous substitutions

393 The rates of adaptive non-synonymous substitutions were estimated with the Grapes program [48],

394 using the Gamma-Exponential DFE, as this model was previously shown to best fit the data [39]. As 395 this method does not accommodate SNPs with missing information in some individuals, we reduced 396 the original data set to $\mathrm{n}=110$ and $\mathrm{n}=105$ genomes for $D$. melanogaster and $A$. thaliana, respectively, 397 by randomly down-sampling sites with at least $\mathrm{n}$ genotypes available and discarding positions where 398 less than $\mathrm{n}$ genotypes were present. Estimates of substitution rates and their confidence intervals were 399 obtained with a bootstrap analysis by sampling genes in each category, with replacement. We 400 performed a total of 100 replicates, and the DFE model was fitted for each replicate with Grapes.

401 Results for $\omega, \omega_{n a}$ and $\omega_{a}$ were plotted using the R package "ggplot2" [99] by taking the mean value and the $95 \%$ confidence interval of the 100 bootstrap replicates performed for each category (see

403 detailed R scripts in the supplementary files in the supplementary data online,

404 https://gitlab.gwdg.de/molsysevol/supplementarydata_geneage). 
Gene age vs. protein length and gene expression

407 Data on protein length and gene expression were included in the datasets of Moutinho et al. [39]

408 (Tables S2 and S3 in supplementary data online). Gene expression data were downloaded from the

409 database Expression Atlas [100], taking one baseline experiment for each species [39]. Mean gene

410 expression levels were then obtained by averaging across samples and tissues for each gene. To

411 correct for these two factors, we divided our dataset into two equally sized groups based on the factor

412 we wished to control. Short proteins had a size up to 366 and 389 amino-acids, and long proteins had a

413 size up to 4,674 and 5,098 amino-acids in A. thaliana and D. melanogaster, respectively. We further

414 merged phylostrata containing a low number of genes. For D. melanogaster, we categorised gene age

415 into 6 main clades by combining clades 3-4, 5-6, 7-10, and 11-12, keeping the others unchanged. In $A$.

416 thaliana, we combined the 15 clades in 6 main groups by merging clades 5-8 and 9-15. For gene

417 expression, we used a total of 17,126 and 6,247 genes for A. thaliana and D. melanogaster,

418 respectively, being categorised as lowly and highly expressed. Genes were classified as lowly

419 expressed if the mean expression levels were up to 10.3 and 6.8, and highly expressed genes were the 420 ones with expression up to 6,632.8 and 4,237.0 in A. thaliana and D. melanogaster, respectively. For

421 D. melanogaster, we categorised gene age in 6 categories by combining clades 3-5, 6-9, and 10-12. In

422 A. thaliana, we combined the data in 6 clades, merging clades 4-7, 8-11 and 12-15.

\section{Gene age vs. protein structure}

425 Since most young genes lack a defined three-dimensional structure [36], they do not have information

426 on the residue's solvent accessibility. Hence, we used a deep learning approach, NetSurfP-2.0, that

427 predicts the RSA of each residue from the amino-acid sequence [101] by using as a training model the

428 HH-suite sequence alignment tool for protein similarity searches [102]. To assess whether this

429 approach provided reliable results, we compared the RSA estimates of NetSurfP-2.0 with those

430 obtained from the PDB structures in our dataset [39]. We found a good correlation between the two

431 approaches for both species (Kendall's $\tau=0.571, \mathrm{p}<2 \mathrm{e}-216 ; \tau=0.462, \mathrm{p}<2 \mathrm{e}-216$, for $D$.

432 melanogaster and $A$. thaliana respectively). Using NetSurfP-2.0, RSA estimates were successfully

433 obtained for a total of 4,238,686 ( $88 \%$ of the total codon sites) and 7,479,807 ( $99 \%$ of the total codon

434 sites) amino-acid residues for D. melanogaster and A. thaliana, respectively. To assess the impact of

435 RSA at the gene level, we analysed the total number of genes in both species by making two

436 categories of genes according to the average RSA value per gene. Genes with lower RSA had mean

437 values between 0.127-0.389 in Drosophila and 0.217-0.386 in Arabidopsis. Genes with a higher RSA

438 had mean values between 0.390-0.894 in Drosophila and 0.387-0.898 in Arabidopsis. The phylostrata 439 groups were defined by combining clades 7-8 in D. melanogaster, and 8-11, 12-15 in A. thaliana.

440 For the analysis on the residue intrinsic disorder, we used the estimates included in the 441 datasets of Moutinho et al. [39]. These data were obtained with the software DisEMBL [103], where

442 intrinsic disorder was estimated per site and classified according to the degree of "hot loops" i.e., 
highly mobile loops. This analysis was performed for 17,732 and 7,410 genes for A. thaliana and $D$. melanogaster, respectively. Genes were combined into two categories according to the mean value of their residue's intrinsic disorder. Genes with a low level of intrinsic disorder had values between 0.029-0.080 in Drosophila and among 0.041-0.084 in Arabidopsis. Genes with a higher degree of intrinsic disorder had values between 0.081-0.554 in Drosophila and among 0.085-0.551 in Arabidopsis. In D. melanogaster, all the 12 phylostrata could be used. In A. thaliana, the 15 strata were combined in 12 categories by merging clades 9-10, 11-12 and 13-14.

\section{Correcting for BLAST e-values}

452 We analysed the robustness of the gene age's effect by correcting the variation in the Expect (E) value 453 estimates in BLAST's searches between our focus species and their respective outgroups. By reducing 454 the variation in E-values estimates, we could correct for potential failures in BLAST's homology searches. To do so, we used a subset of genes for which the correlation between the $\mathrm{E}$ value and gene age was no longer significant: 12,472 genes with an E value lower than 1e-150 for A. thaliana and 7,104 genes with an E value lower than 1e-100 for D. melanogaster (supplementary file S2). For $A$. thaliana, analyses were carried by combining clades 8-13, with no genes left in clades 14 and 15 . For D. melanogaster, analyses were performed with the 12 strata.

Gene age vs. protein function

462 Gene ontology terms were obtained from the "dmelanogaster_gene_ensembl" and the

463 "athaliana_eg_gene" tables in the Ensembl database (version 103), through the R package "biomaRt"

464 [104]. A total of 7,253 ( 70\% of the genes) and 15,604 ( $80 \%$ of the genes) genes were mapped in $D$. melanogaster and A. thaliana, respectively. To check whether the effect of gene age prevailed across functional protein classes, we analysed the GO terms with the highest number of young genes mapped: more than 50 genes present in Clades 11 and 12 in D. melanogaster; and more than 30 genes present in Clades 12 to 15 in A. thaliana. This filtering step resulted in 6,637 genes across 23 GO categories in D. melanogaster (Table S2 in Supplementary Data online), and 15,410 genes across 10 GO categories in A. thaliana (Table S3 in Supplementary Data online). To analyse the effect of gene age, we compared three age classes. In D. melanogaster, the first age category spanned over phylostrata $1-3$, the second category covered clades $4-7$, and the third one included clades $8-12$. In $A$. thaliana, the first category comprised genes belonging to clades 1-6, the second category spanned over clades 7-11, and the third one included the phylostrata between clades 12-15 (Figure 1a).

477 We obtained PPI data for D. melanogaster from the STRING database [105], which includes both 478 physical and functional interactions (https://string-db.org/). This database included 13,046 proteins 
with annotated interactions, which were used to analyse the distribution of protein networks across phylostrata.

\section{The fitness effects of amino-acid substitutions}

483 We used Grantham's physicochemical distances between amino-acids [67] as a proxy for the fitness effects of amino-acid substitutions and performed two analyses. In the first one, we calculated the average Grantham's distance between amino acid substitutions for each phylostrata. In the second analysis, we estimated the average Grantham's distance for adaptive $\left(\bar{G}_{a}\right)$ and non-adaptive $\left(\bar{G}_{n a}\right)$ non-synonymous substitutions in each age stratum. We restricted our analysis to amino acid pairs separated by a single mutational step. To estimate the rate of adaptive and non-adaptive nonsynonymous amino acid substitutions between pairs of amino acids we used the same approach as Bergman and Eyre-Walker [106]. This method compares the non-synonymous SFS for a particular amino acid pair, for example alanine and aspartic acid, which are separated by a $\mathrm{A}<\mathrm{C}$ mutation, with the synonymous SFS of 4-fold degenerate sites separated only by $\mathrm{A}<\mathrm{C}$ mutations $\left(S F S_{4 F(A<>C)}\right)$. For amino acid pairs separated by more than one mutation type, we estimated the

494 weighted average of the synonymous SFS of 4-fold sites for the different types of mutations, weighting by the frequency of the respective codons. For example, serine and cysteine are separated by $\mathrm{A}<>\mathrm{T}$ and $\mathrm{C}<>\mathrm{G}$ mutations. The synonymous SFS for this amino acid pair was estimated as $S F S_{4 F(\text { weighted })}=\left[\left(f_{A G T}+f_{T G T}+f_{A G C}+f_{T G C}\right) * S F S_{4 F(A<>T)}+\left(f_{T C T}+f_{T G T}+f_{T C C}+f_{T G C}\right) *\right.$ $\left.S F S_{4 F(C<>G)}\right] /\left(f_{A G T}+f_{T G T}+f_{A G C}+f_{T G C}+f_{T G T}+f_{T G C}\right)$. It is not possible to estimate the rates of adaptive and non-adaptive substitution for each pair of amino acids for each phylostratum because we have insufficient data. So, we combined amino acid pairs into ten categories based on their Grantham's distance, and we combined clades 5-6, 8-11 and 12-15 in Arabidopsis, and clades 3-4, 5-7 and 8-10 in Drosophila. We used Grapes to estimate $\omega_{a}$ and $\omega_{n a}$ for the ten categories of Grantham's physicochemical distances for each combined age stratum. We calculated the average Grantham's distance for adaptive $\left(\bar{G}_{a}\right)$ and non-adaptive $\left(\bar{G}_{n a}\right)$ non-synonymous substitutions in each age stratum as $\bar{G}_{a}=\sum_{i} \omega_{a i} \bar{g}_{l} N_{i} / \sum_{i} N_{i}$ and $\bar{G}_{n a}=\sum_{i} \omega_{n a i} \bar{g}_{l} N_{i} / \sum_{i} N_{i}$ where the sum is across the groups of amino acids grouped by Grantham's distance, $\omega_{a i}$ and $\omega_{\text {nai }}$ are the rates of adaptive and nonadaptive substitution in the ith group, $\overline{g_{l}}$ is the average grantham's distance amongst the amino acid pairs in the group and $N_{i}$ is the number of non-synonymous sites in the $i$ th group.

\section{$\underline{\text { Statistical analyses }}$}

511 Assessing the effect of gene age within each protein functional class was performed by comparing rate

512 estimates between all pairs of age categories. 100 bootstrap replicates were generated and $\omega_{a}$ and $\omega_{n a}$

513 were estimated for each resampling, allowing to compute the rate differences between categories. A

514 one-tailed $\mathrm{P}$-value can be obtained using the formula $\mathrm{P}=(2 \mathrm{k}+1) /(\mathrm{N}+1)$, where $\mathrm{N}=100$ is the

515 number of bootstrap replicates and $\mathrm{k}$ is the number of times the computed difference was greater (resp. 
516 lower) than 0 . Here, we used a two-tailed version of this test, computing the $\mathrm{P}$-value as $\mathrm{P}=[2 * \min$

$\left.517\left(\mathrm{k}^{-}, \mathrm{k}^{+}\right)+1\right] /(\mathrm{N}+1)$, where $\mathrm{k}^{-}$is the number of times the difference was negative, and $\mathrm{k}^{+}$is the number

518 of times the difference was positive. P-values for all pairwise comparisons were corrected for multiple

519 testing using the FDR method [107] as implemented in R [108] (see detailed R script in supplementary

520 file S3). For the analysis with PPI and gene age, statistical significance was assessed using non-

521 parametric posthoc tests, as implemented in the "Kruskal" method of the R package "agricolae" using

522 the FDR method to correct for multiple testing [109] (see detailed R script in supplementary file S5).

523 For the rest of the analyses, statistical significance was assessed with Kendall's correlation tests using

524 the mean value of the 100 bootstrap replicates for each category (see detailed script in supplementary

525 file S6). To estimate the combined P-value for each co-factor we used the weighted-Z method using

526 the R package "metap" [110]. We estimated the weight of each p-value using a linear modelling

527 approach with $\omega_{a}$ and $\omega_{n a}$ as response variables, and gene age and potential co-factors as explanatory

528 variables and inferred the reciprocal of the squared standard error of the residuals in each model (see

529 detailed R scripts in supplementary file S7). To determine whether the chromosome impacted gene

530 age's effect on estimates of $\omega_{a}$ and $\omega_{n a}$, we performed an analysis of covariance (ANCOVA) by

531 comparing a model M1 that included the impact of the chromosome, age, and their interaction, with a

532 model M0 that included age only (see detailed R script in supplementary file S1). Normality,

533 homoscedasticity, and independence of the error terms of the model were assessed with the package

534 "Imtest" [111] in R. Finally, the MK regression analysis was performed per site, where we analysed

535 the combined effect of RSA, intrinsic protein disorder, protein length, gene expression, protein

536 function and the sex chromosome in Drosophila. As this method does not deal with categorical

537 variables, we created a binary variable for protein function based on the gene's presence (1.0) or

538 absence (0.0) in stress-related proteins (i.e., response to stress, response to external stimulus and signal

539 transduction). We used the same rationale for the chromosome X effect in Drosophila. We performed

540 the MK regression analysis by comparing polymorphic and divergence sites between non-synonymous

541 and 4-fold degenerated sites in both species. As this method does not account for the effects of weak

542 selection [57], we analysed only sites with an allele frequency above $50 \%$.

544 Acknowledgments

545 The authors thank Diethard Tautz, Tal Dagan and Chaitanya Gokhale for fruitful discussions. J.Y.D.

546 acknowledges funding from the Max Planck Society.

\section{$548 \quad$ References}

549 1. Fisher R. The Genetical Theory of Natural Selection. Oxford Univ. Press, Oxford; 1930.

550 2. Wright S. Evolution in Mendelian Populations. Genetics. 1931;16(2):97-159.

5513 . Wright $\mathrm{S}$. The roles of mutation, inbreeding, crossbreeding and selection in evolution. Vol. 1,

$552 \quad$ Sixth International Congress on Genetics. 1932. p. 356-66. 
4. Smith JM. Natural selection and the concept of a protein space. Nature. 1970;225(5232):563-4.

5. Gillespie JH. A Simple Stochastic Gene Substitution Model. Theor Popul Biol. 1983;23:20215.

556 6. Gillespie JH. Molecular evolution over the mutational landscape. Evolution. 1984;38(5):111629.

7. Gillespie JH. The Causes of Molecular Evolution. Oxford University Press; 1991.

559 8. Kimura M. The Neutral Theory of Molecular Evolution. Cambridge Univeristy Press; 1983.

560 9. Orr HA. The Population Genetics of Adaptation: The Distribution of Factors Fixed during Adaptive Evolution. Evolution. 1998;52(4):935.

10. Orr AH. The evolutionary genetics of adaptation: A simulation study. Genet Res. 1999;74(3):207-14.

564 11. Lenski RE, Rose MR, Simpson SC, Tadler SC. Long-Term Experimental Evolution in Escherichia coli. I. Adaptation and Divergence During 2,000 Generations. Am Nat. 1991;138(6):1315-41.

12. Cooper VS, Lenski RE. The population genetics of ecological specialization in evolving Escherichia coli populations. Nature. 2000;407(6805):736-9.

13. Gerrish P. The rhythm of microbial adaptation. Nature. 2001;413(6853):299-302.

14. Rozen DE, De Visser JAGM, Gerrish PJ. Fitness effects of fixed beneficial mutations in microbial populations. Curr Biol. 2002;12(12):1040-5.

15. Schoustra SE, Bataillon T, Gifford DR, Kassen R. The properties of adaptive walks in evolving populations of fungus. PLoS Biol. 2009;7(11): e1000250.

574 16. Lynch M. Genomics: Gene duplication and evolution. Science. 2002;297(5583):945-7.

17. Tautz D, Domazet-Lošo T. The evolutionary origin of orphan genes. Nat Rev Genet. 2011;12(10):692-702.

18. Cohen O, Ashkenazy H, Belinky F, Huchon D, Pupko T. GLOOME: Gain loss mapping engine. Bioinformatics. 2010;26(22):2914-5.

19. Altschul S, Madden T, Schaffer A, Zhang J, Zhang Z, Miller W, et al. Gapped blast and psiblast: a new generation of protein database search programs. FASEB J. 1998;12(8):3389-402.

20. Domazet-Lošo T, Brajković J, Tautz D. A phylostratigraphy approach to uncover the genomic history of major adaptations in metazoan lineages. Trends Genet. 2007;23(11):533-9.

21. Thornton K, Long M. Rapid divergence of gene duplicates on the Drosophila melanogaster X chromosome. Mol Biol Evol. 2002;19(6):918-25.

22. Cai JJ, Petrov DA. Relaxed purifying selection and possibly high rate of adaptation in primate lineage-specific genes. Genome Biol Evol. 2010;2(1):393-409.

23. Domazet-Loso T, Tautz D. An evolutionary analysis of orphan genes in Drosophila. Genome Res. 2003;13(10):2213-9.

24. Vishnoi A, Kryazhimskiy S, Bazykin GA, Hannenhalli S, Plotkin JB. Young proteins 
experience more variable selection pressures than old proteins. Genome Res. 2010;20(11):1574-81.

25. Cai JJ, Woo PCY, Lau SKP, Smith DK, Yuen KY. Accelerated evolutionary rate may be responsible for the emergence of lineage-specific genes in Ascomycota. J Mol Evol. 2006;63(1):1-11.

26. Wolf YI, Novichkov PS, Karev GP, Koonin E V., Lipman DJ. The universal distribution of evolutionary rates of genes and distinct characteristics of eukaryotic genes of different apparent ages. Proc Natl Acad Sci. 2009;106(18):7273-80.

27. Albà MM, Castresana J. Inverse relationship between evolutionary rate and age of mammalian genes. Mol Biol Evol. 2005;22(3):598-606.

28. Domazet-Lošo T, Carvunis AR, Albà MM, Šestak MS, Bakarić R, Neme R, et al. No evidence for phylostratigraphic bias impacting inferences on patterns of gene emergence and evolution. Mol Biol Evol. 2017;34(4):843-56.

29. Zhang YE, Vibranovski MD, Krinsky BH, Long M. Age-dependent chromosomal distribution of male-biased genes in Drosophila. Genome Res. 2010;20(11):1526-33.

30. Daubin V, Ochman H. Bacterial genomes as new gene homes: The genealogy of ORFans in E. coli. Genome Res. 2004;14(6):1036-42.

32. Cui X, Lv Y, Chen M, Nikoloski Z, Twell D, Zhang D. Young genes out of the male: An insight from evolutionary age analysis of the pollen transcriptome. Mol Plant. 2015;8(6):93545.

33. Arendsee ZW, Li L, Wurtele ES. Coming of age: Orphan genes in plants. Trends Plant Sci. 2014;19(11):698-708.

34. Kuo CH, Kissinger JC. Consistent and contrasting properties of lineage-specific genes in the apicomplexan parasites Plasmodium and Theileria. BMC Evol Biol. 2008;8(1):1-16.

35. Neme R, Tautz D. Phylogenetic patterns of emergence of new genes support a model of frequent de novo evolution. BMC Genomics. 2013;14(1):1-13.

36. Wilson BA, Foy SG, Neme R, Masel J. Young genes are highly disordered as predicted by the ribonuclease gene in a leaf-eating monkey. Nat Genet. 2002;30(4):411-5.

624 39. Moutinho AF, Trancoso FF, Dutheil JY. The impact of protein architecture on adaptive evolution. Mol Biol Evol. 2019;36(9): 2013-2028.

626 40. Afanasyeva A, Bockwoldt M, Cooney CR, Heiland I, Gossmann TI. Human long intrinsically 
disordered protein regions are frequent targets of positive selection. Genome Res. 2018;28(7):975-82.

629 41. Subramanian S, Kumar S. Gene expression intensity shapes evolutionary rates of the proteins encoded by the vertebrate genome. Genetics. 2004;168(1):373-81.

42. Stukenbrock EH, Bataillon T, Dutheil JY, Hansen TT, Li R, Zala M, et al. The making of a new pathogen: Insights from comparative population genomics of the domesticated wheat pathogen Mycosphaerella graminicola and its wild sister species. Genome Res. 2011;21(12):2157-66.

43. Enard D, Cai L, Gwennap C, Petrov DA. Viruses are a dominant driver of protein adaptation in mammals. Elife. 2016;5: e12469.

44. Rocha EPC, Danchin A. An Analysis of Determinants of Amino Acids Substitution Rates in Bacterial Proteins. Mol Biol Evol. 2004;21(1):108-16.

45. Pal C, Papp B, Hurst LD. Highly Expressed Genes in Yeast Evolve Slowly. Genetics. 2001;158(1998):927-31.

46. Sackton TB, Lazzaro BP, Schlenke TA, Evans JD, Hultmark D, Clark AG. Dynamic evolution of the innate immune system in Drosophila. Nat Genet. 2007;39(12):1461-8.

47. Obbard DJ, Welch JJ, Kim KW, Jiggins FM. Quantifying adaptive evolution in the Drosophila immune system. PLoS Genet. 2009;5(10): e1000698.

48. Galtier N. Adaptive Protein Evolution in Animals and the Effective Population Size Hypothesis. PLoS Genet. 2016;12(1):1-23.

49. Whitlock MC, Whitlock MC. Combining probability from independent tests: the weighted Zmethod is superior to Fisher's approach. Wiley Online Libr. 2005;18(5):1368-73.

50. Vicoso B, Charlesworth B. Evolution on the X chromosome: Unusual patterns and processes. Nat Rev Genet. 2006;7(8):645-53.

51. Vicoso B, Charlesworth B. Effective population size and the faster-X effect: An extended model. Evolution. 2009;63(9):2413-26.

52. Charlesworth B, Campos JL, Jackson BC. Faster-X evolution: Theory and evidence from Drosophila. Mol Ecol. 2018;27(19):3753-71.

53. Stoletzki N, Eyre-Walker A. Estimation of the neutrality index. Mol Biol Evol. 2011;28(1):6370.

54. Smith NGC, Eyre-Walker a. Adaptive protein evolution in Drosophila . Nature. 2002; 415(6875):1022-1024.

55. Ding Y, Zhou Q, Wang W. Origins of New Genes and Evolution of Their Novel Functions.

Annu Rev Ecol Evol Syst. 2012;43(1):345-63.

661 56. Choi SS, Vallender EJ, Lahn BT. Systematically assessing the influence of 3-dimensional structural context on the molecular evolution of mammalian proteomes. Mol Biol Evol. 
57. Huang Y-F. Dissecting Genomic Determinants of Positive Selection with an Evolution-Guided Regression Model. Mol Biol Evol. 2021(msab291).

58. Eyre-Walker A, Keightley PD. Estimating the rate of adaptive molecular evolution in the presence of slightly deleterious mutations and population size change. Mol Biol Evol. 2009;26(9):2097-108.

59. Elhaik E, Sabath N, Graur D. The "inverse relationship between evolutionary rate and age of mammalian genes" is an artifact of increased genetic distance with rate of evolution and time of divergence. Mol Biol Evol. 2006;23(1):1-3.

60. Moyers BA, Zhang J. Phylostratigraphic bias creates spurious patterns of genome evolution. Mol Biol Evol. 2015;32(1):258-67.

61. Moyers BA, Zhang J. Evaluating Phylostratigraphic Evidence for Widespread de Novo Gene Birth in Genome Evolution. Mol Biol Evol. 2016;33(5):1245-56.

62. Albà MM, Castresana J. On homology searches by protein Blast and the characterization of the age of genes. BMC Evol Biol. 2007;7(1):1-8.

63. Khalturin K, Hemmrich G, Fraune S, Augustin R, Bosch TCG. More than just orphans: are taxonomically-restricted genes important in evolution? Trends Genet. 2009;25(9):404-13. of protein evolution and polymorphism in arabidopsis. Genome Biol Evol. 2011;3(1):1210-9.

65. Geldner N, Robatzek S. Plant receptors go endosomal: A moving view on signal transduction. Plant Physiol. 2008;147(4):1565-74.

684 66. Groen AJ, De Vries SC, Lilley KS. A proteomics approach to membrane trafficking. Plant Physiol. 2008;147(4):1584-9.

67. Grantham R. Amino Acid Difference Formula to Help Explain Protein Evolution. Science. 1974;185(4154):862-4.

68. Castellano D, Coronado-Zamora M, Campos JL, Barbadilla A, Eyre-Walker A. Adaptive evolution is substantially impeded by hill-Robertson interference in drosophila. Mol Biol Evol. 2016;33(2):442-55.

69. Hoffman PF, Kaufman AJ, Halverson GP, Schrag DP. A neoproterozoic snowball earth.

693 70. Montooth KL, Marden JH, Clark AG. Mapping Determinants of Variation in Energy Metabolism, Respiration and Flight in Drosophila. Genetics. 2003;165(2):623-35.

71. Paixão T, Barton NH. The effect of gene interactions on the long-term response to selection. Proc Natl Acad Sci. 2016;113(16):4422-7. 2013;67(12):3501-11. 
Sci. 2018; 285(1870): 20172599.

74. Lagator M, Colegrave N, Neve P. Selection history and epistatic interactions impact dynamics of adaptation to novel environmental stresses. Proc R Soc B Biol Sci. 2014;281(1794).

75. Wang H, Moore MJ, Soltis PS, Bell CD, Brockington SF, Alexandre R, et al. Rosid radiation and the rapid rise of angiosperm-dominated forests. Proc Natl Acad Sci. 2009;106(10):3853-8.

76. Ohno S. Evolution by gene duplication. Springer Science \& Business Media; 1970.

77. Piatigorsky J, Wistow G. The recruitment of crystallins: new functions precede gene duplication. Science. 1991;252(5009):1078-9.

78. Force A, Lynch M, Pickett FB, Amores A, Yan YL, Postlethwait J. Preservation of duplicate genes by complementary, degenerative mutations. Genetics. 1999;151(4):1531-45.

79. Prince VE, Pickett FB. Splitting pairs: The diverging fates of duplicated genes. Nat Rev Genet. 2002;3(11):827-37.

713 80. Jacob F. Evolution and Tinkering. Science. 1977;196(4295):1161-6.

714 81. Cai J, Zhao R, Jiang H, Wang W. De novo origination of a new protein-coding gene in Saccharomyces cerevisiae. Genetics. 2008;179(1):487-96.

82. Heinen TJAJ, Staubach F, Häming D, Tautz D. Emergence of a New Gene from an Intergenic Region. Curr Biol. 2009;19(18):1527-31.

83. Zhao L, Saelao P, Jones CD, Begun DJ. Origin and spread of de novo genes in Drosophila melanogaster populations. Science. 2014;343(6172):769-72.

84. Neme R, Tautz D. Evolution: Dynamics of de novo gene emergence. Curr Biol. 2014;24(6):R238-40.

85. Palmieri N, Kosiol C, Schlötterer C. The life cycle of Drosophila orphan genes. Elife. 2014;3: e01311.

86. Carvunis AR, Rolland T, Wapinski I, Calderwood MA, Yildirim MA, Simonis N, et al. Protogenes and de novo gene birth. Nature. 2012;487(7407):370-4.

87. Smith JM. Natural selection and the concept of a protein space. Nature. 1970;225:563-4.

88. Orr HA. the Population Genetics of Adaptation: the Adaptation of Dna Sequences. Evolution. 2002;56(7):1317.

89. Bustamante CD, Nielsen R, Sawyer SA, Olsenk KM, Purugganank MD, Hartl DL. The Cost of Inbreeding in Arabidopsis. Nature. 2002; 416(6880), 531-534.

90. Charlesworth B, Morgan MT, Charlesworth D. The effect of deleterious mutations on neutral molecular variation. Genetics. 1993;134(4):1289-303.

91. Assaf ZJ, Petrov DA, Blundell JR. Obstruction of adaptation in diploids by recessive, strongly between advantageous and deleterious variants. Elife. 2021;10:e69026. 
93. Gossmann TI, Keightley PD, Eyre-Walker A. The effect of variation in the effective population size on the rate of adaptive molecular evolution in eukaryotes. Genome Biol Evol. 2012;4(5):658-67.

94. Eyre-Walker A. The genomic rate of adaptive evolution. Trends Ecol Evol. 2006;21(10):569575.

95. Rousselle M, Simion P, Tilak M-K, Figuet E, Nabholz B, Galtier N. Is adaptation limited by mutation? A timescale-dependent effect of genetic diversity on the adaptive substitution rate in animals. PLOS Genet. 2020;16(4):e1008668.

96. Pool JE, Corbett-Detig RB, Sugino RP, Stevens KA, Cardeno CM, Crepeau MW, et al. Population Genomics of Sub-Saharan Drosophila melanogaster: African Diversity and NonAfrican Admixture. PLoS Genet. 2012;8(12): e1003080.

97. Weigel D, Mott R. The 1001 Genomes Project for Arabidopsis thaliana. Genome Biol. 2009; $10(5): 1-5$.

98. Guéguen L, Gaillard S, Boussau B, Gouy M, Groussin M, Rochette NC, et al. Bio++: Efficient extensible libraries and tools for computational molecular evolution. Mol Biol Evol. 2013;30(8):1745-50.

99. Wickham H. ggplot2 - Elegant Graphics for Data Analysis (2nd Edition). J Stat Softw. 2017;77:2-5.

100. Petryszak R, Keays M, Tang YA, Fonseca NA, Barrera E, Burdett T, et al. Expression Atlas update - An integrated database of gene and protein expression in humans, animals and plants. Nucleic Acids Res. 2016; 44(D1): D746-D752.

101. Klausen MS, Jespersen MC, Nielsen H, Jensen KK, Jurtz VI, Sønderby CK, et al. NetSurfP2.0: Improved prediction of protein structural features by integrated deep learning. Proteins Struct Funct Bioinforma. 2019;87(6):520-7.

102. Remmert M, Biegert A, Hauser A, Söding J. HHblits: Lightning-fast iterative protein sequence searching by HMM-HMM alignment. Nat Methods. 2012;9(2):173-5.

103. Linding R, Jensen LJ, Diella F, Bork P, Gibson TJ, Russell RB. Protein disorder prediction: Implications for structural proteomics. Structure. 2003;11(11): 1453-1459.

104. Durinck S, Moreau Y, Kasprzyk A, Davis S, De Moor B, Brazma A, et al. BioMart and Bioconductor: A powerful link between biological databases and microarray data analysis. Bioinformatics. 2005;21(16):3439-40.

105. Jensen LJ, Kuhn M, Stark M, Chaffron S, Creevey C, Muller J, et al. STRING 8 - A global view on proteins and their functional interactions in 630 organisms. Nucleic Acids Res. 2009;37(SUPPL. 1): D412-D416.

106. Bergman J, Eyre-Walker A. Does Adaptive Protein Evolution Proceed by Large or Small Steps at the Amino Acid Level? Mol Biol Evol. 2019; 36(5):990-998..

107. Benjamini Y, Hochberg Y. Controlling the False Discovery Rate : A Practical and Powerful 
Approach to Multiple Testing. J R Stat Soc. 1995;57(1):289-300.

776 108. R Core Team. A language and environment for statistical computing. R Foundation for Statistical Computing, Vienna, Austria. 2017.

778 109. Mendiburu F, Simon R, De Mendiburu F. Agricolae-Ten years of an Open source Statistical tool for experiments in Breeding, agriculture and biology. PeerJ PrePrints. 2015.

780 110. Dewey M. metap: meta-analysis of significance values. R package version 1.4. 2020;

781 111. Zeileis A, Hothorn T. Diagnostic checking in regression relationships.. R News. 2002;2(3):7782 10.

783 


\section{Figures}

786

(a)
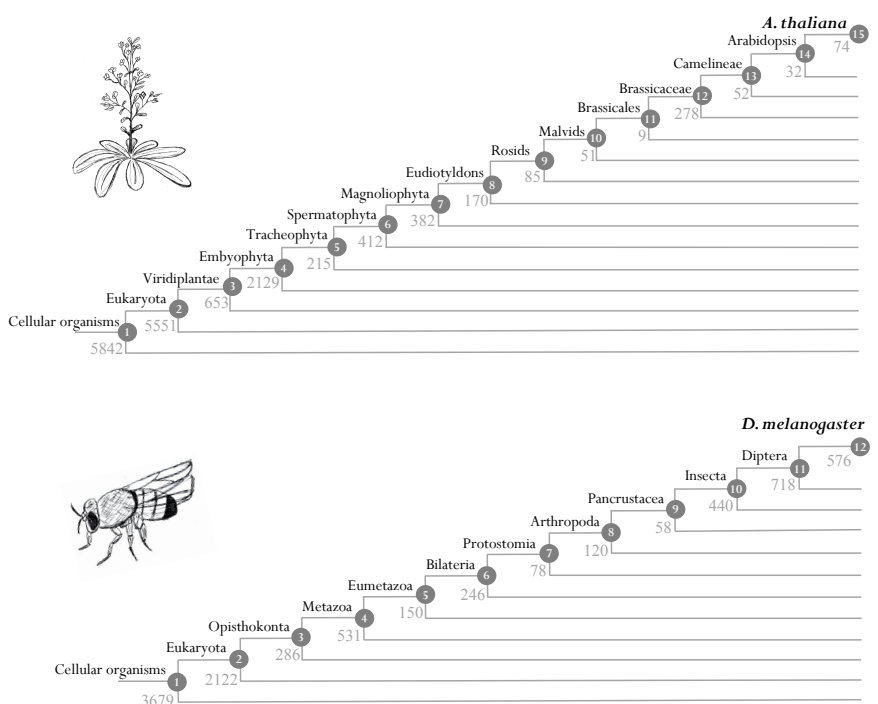

787

788 Figure 1. (a) Phylogenetic definition of the strata used in the analyses for $A$. thaliana (top) and $D$.

melanogaster (bottom). The number of genes mapped to each clade is shown. (b) Relationship

790 between the rate of protein evolution $(\omega)$, non-adaptive non-synonymous substitutions $\left(\omega_{n a}\right)$ and

791 adaptive non-synonymous substitutions $\left(\omega_{a}\right)$ with gene age in $A$. thaliana (top) and in $D$.

792 melanogaster (bottom). Clades are ordered according to (a). In D. melanogaster, the results for X-

793 linked, autosomal, and total genes are shown. Mean values of $\omega, \omega_{n a}$ and $\omega_{a}$ for each category are

794 represented with the black points. Error bars denote for the $95 \%$ confidence interval for each category,

795 computed over 100 bootstrap replicates.

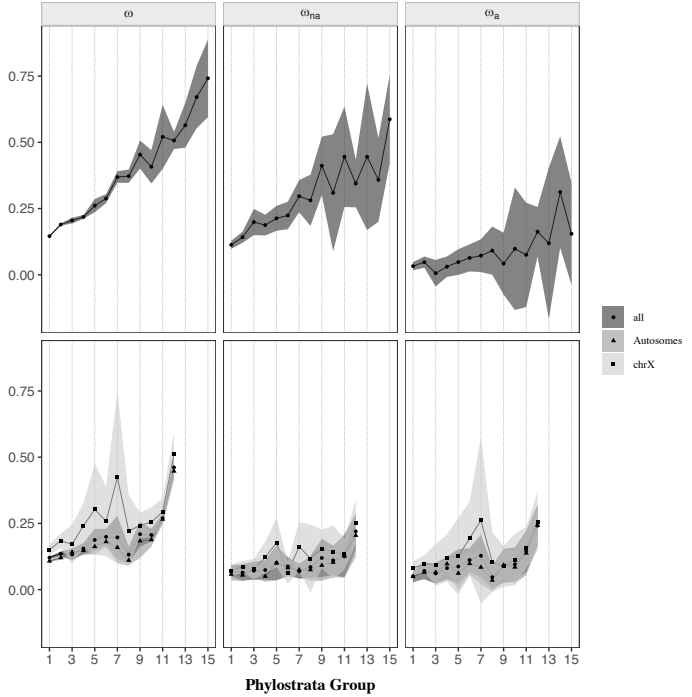


(a)

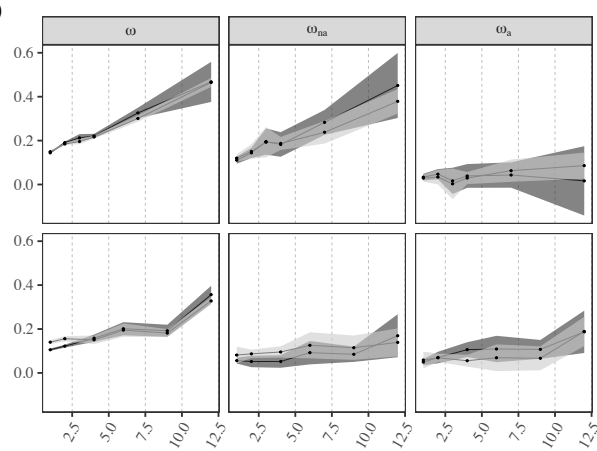

(c)

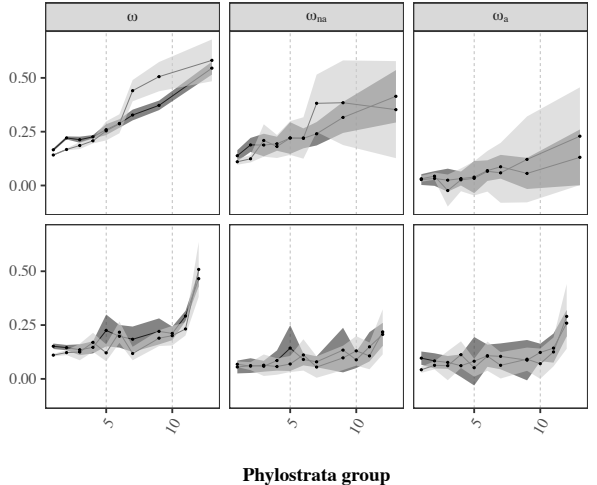

(b)

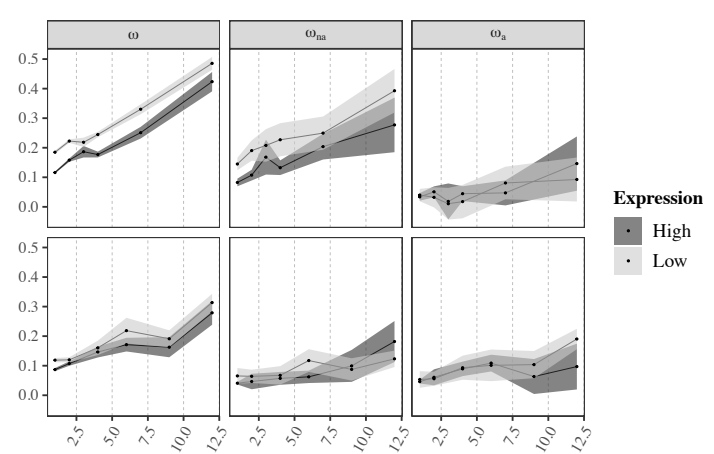

(d)

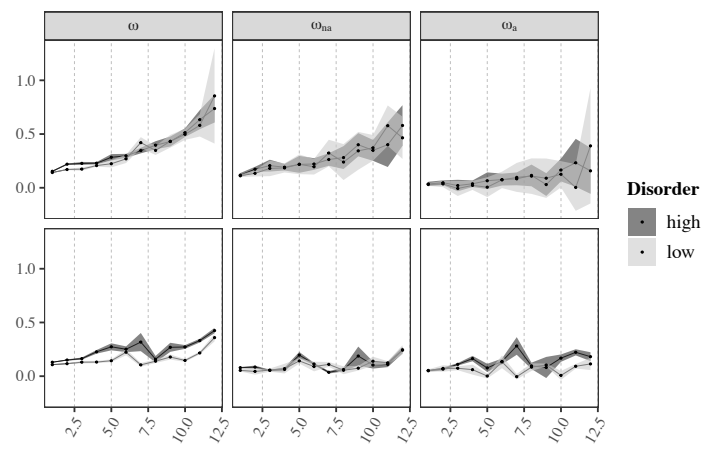

Phylostrata group

798 Figure 2. Estimates of $\omega, \omega_{n a}$ and $\omega_{a}$ plotted as a function of (a) protein length and (b) mean

799 expression levels, (c) relative solvent accessibility, and (d) protein intrinsic disorder with gene age in

800 A. thaliana (top) and D. melanogaster (bottom). Analyses were performed by comparing short and

801 long genes (a), lowly and highly expressed genes (b), proteins with low and high mean RSA values

802 (c), and proteins with low and high average intrinsic disorder (d) across age categories (see Material

803 and Methods). Legend as in Figure 1. 

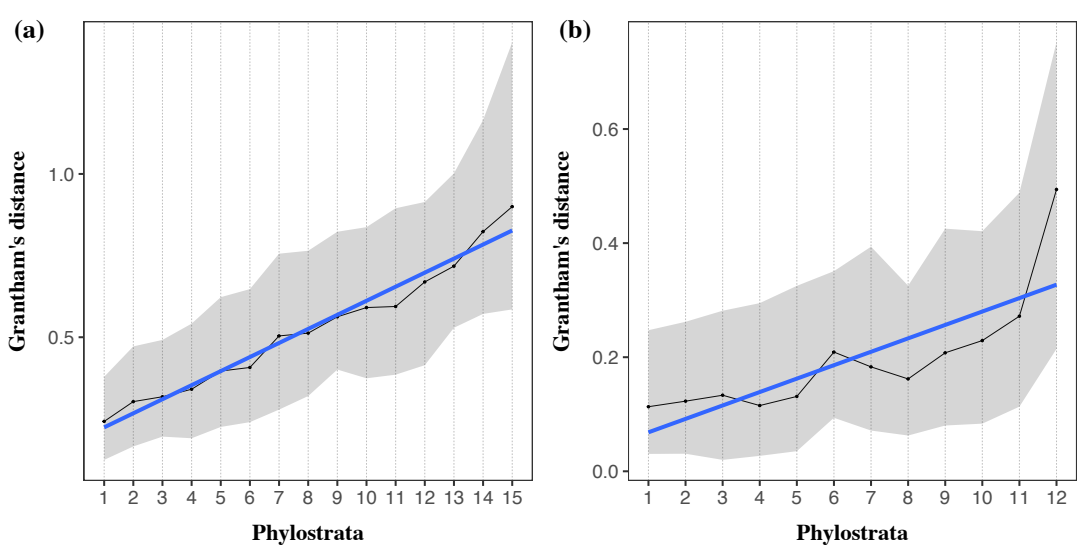

Figure 3. Relationship between gene age and Grantham's distance between amin-acids for A. thaliana (a) and D. melanogaster (b). A linear model was fitted between gene age and Grantham's distances

808 values and is represented with the blue line. For each clade, the median value of the Grantham's distance

809 between residues is depicted with the black dot. The shaded area represents the physicochemical distances within the $1^{\text {st }}$ and $3^{\text {rd }}$ quartile. 
Table 1. Kendall's correlation coefficients for the relationship between $\omega, \omega_{n a}$ and $\omega_{a}$ and gene age, for the analysis of gene age and the combined analyses of gene age with the respective co-factors: protein length, gene expression, protein intrinsic disorder and the mean relative solvent accessibility per gene. The combined probabilities for each co-factor within and across species are presented in the fields "Weighted Z" and "Weighted Z across species", respectively, for

\begin{tabular}{|c|c|c|c|c|c|c|c|c|c|c|}
\hline & & \multicolumn{3}{|c|}{ Arabidopsis } & \multicolumn{3}{|c|}{ Drosophila } & \multicolumn{3}{|c|}{ Weighted $Z$ across species } \\
\hline & & $\omega$ & $\omega_{n a}$ & $\omega_{a}$ & $\omega$ & $\omega_{n a}$ & $\omega_{a}$ & $\omega$ & $\omega_{n a}$ & $\omega_{a}$ \\
\hline Gene Age & & $0.962^{* * *}$ & $0.848^{* * *}$ & $0.733^{* * *}$ & $0.727^{* * *}$ & $0.697^{* *}$ & $0.636^{* *}$ & & & \\
\hline \multirow{3}{*}{ Protein Length } & Long & $1.000^{* *}$ & $0.867^{*}$ & -0.200 & $0.867^{*}$ & $0.600^{(.)}$ & $0.867^{*}$ & \multirow{3}{*}{$1.56 \mathrm{e}-04$} & \multirow{3}{*}{$7.71_{* * *}^{\mathrm{e}-05}$} & \multirow{3}{*}{$\underset{* *}{7.98 \mathrm{e}-03}$} \\
\hline & Short & $1.000^{* *}$ & $0.867^{*}$ & $0.600^{(.)}$ & $0.733^{*}$ & $0.867^{*}$ & 0.467 & & & \\
\hline & Weighted Z & $6.46 \mathrm{e}-04^{* * *}$ & $1.61 \mathrm{e}-03^{* *}$ & 0.133 & $2.64 \mathrm{e}-03^{* *}$ & $5.29 \mathrm{e}-03^{* *}$ & $0.0105^{*}$ & & & \\
\hline \multirow{3}{*}{$\begin{array}{l}\text { Gene } \\
\text { Expression }\end{array}$} & High & $0.867^{*}$ & $0.867^{*}$ & 0.467 & $0.867^{*}$ & $1.000^{* *}$ & $0.600^{(.)}$ & \multirow{3}{*}{$\underset{* * *}{6.93 e-05}$} & \multirow{3}{*}{$\underset{* * *}{6.89 \mathrm{e}-06}$} & \multirow{3}{*}{$\underset{* *}{3.53 \mathrm{e}-03}$} \\
\hline & Low & $0.867^{*}$ & $1.000^{* *}$ & 0.333 & $0.867^{*}$ & $0.733^{*}$ & $1.000^{* *}$ & & & \\
\hline & Weighted Z & $1.51 \mathrm{e}-03^{* *}$ & $3.71 \mathrm{e}-04^{* * *}$ & 0.186 & $1.09 \mathrm{e}-03^{* *}$ & $1.68 \mathrm{e}-03^{* *}$ & $2.24 \mathrm{e}-03^{* *}$ & & & \\
\hline \multirow{3}{*}{$\begin{array}{l}\text { Protein } \\
\text { Intrinsic } \\
\text { Disorder }\end{array}$} & High & $1.000^{* * *}$ & $0.939^{* * *}$ & $0.636^{* *}$ & $0.670^{* *}$ & 0.303 & $0.515^{*}$ & \multirow{3}{*}{$\underset{* * *}{2 \mathrm{e}-216}$} & \multirow{3}{*}{$\underset{* * * *}{6.60 \mathrm{e}-06}$} & \multirow{3}{*}{$\underset{* *}{2.53 \mathrm{e}-03}$} \\
\hline & Low & $0.970^{* * *}$ & $0.909^{* * *}$ & $0.454^{*}$ & $0.630^{* *}$ & $0.576^{* *}$ & 0.273 & & & \\
\hline & Weighted Z & $<2 \mathrm{e}-216^{* * *}$ & $<2 \mathrm{e}-216^{* * *}$ & $1.20 \mathrm{e}-03^{* *}$ & $3.85 \mathrm{e}-05^{* * *}$ & $5.80 \mathrm{e}-03^{* *}$ & $4.18 \mathrm{e}-02^{*}$ & & & \\
\hline \multirow{3}{*}{$\begin{array}{l}\text { Mean Relative } \\
\text { Solvent } \\
\text { Accessibility }\end{array}$} & High & $0.944^{* * *}$ & $0.889^{* * *}$ & $0.722^{* *}$ & $0.636^{* *}$ & $0.673^{* *}$ & $0.564^{*}$ & \multirow{3}{*}{$\underset{* * *}{1.00 \mathrm{e}-07}$} & \multirow{3}{*}{$9.00 \mathrm{e}-07$} & \multirow{3}{*}{$1.37 \mathrm{e}-05$} \\
\hline & Low & $1.000^{* * *}$ & $0.778 * *$ & $0.667^{*}$ & $0.636^{* *}$ & $0.491^{*}$ & $0.564^{*}$ & & & \\
\hline & Weighted Z & $6.20 \mathrm{e}-06^{* * *}$ & $1.41 \mathrm{e}-05^{* * *}$ & $1.24 \mathrm{e}-03^{* *}$ & $3.67 \mathrm{e}-04^{* * *}$ & $7.76 \mathrm{e}-04^{* * *}$ & $1.55 \mathrm{e}-03^{* *}$ & & & \\
\hline
\end{tabular}

